\title{
UTILIZAÇÃO DAS TECNOLOGIAS DA INDÚSTRIA 4.0 NA SEGURANÇA E SAÚDE DO TRABALHADOR: UMA REVISÃO SISTEMÁTICA DA LITERATURA
}

\author{
USE OF INDUSTRY 4.0 TECHNOLOGIES IN OCCUPATIONAL HEALTH AND SAFETY: A \\ SYSTEMATIC LITERATURE REVIEW
}

\section{Caroline Martirena Monks da Silva ${ }^{1}$, Marco Antonio Garcez da Silva ${ }^{2}$, Aline Soares Pereira ${ }^{3}$, Luis Antonio dos Santos Franz ${ }^{4}$, \& Renata Heidtmann-Bemvenuti ${ }^{* 5}$ 12345 Universidade Federal de Pelotas (UFPEL), Pelotas, RS, Brasil. \\ ${ }^{1}$ carolinemmonks@gmail.com ${ }^{2} \underline{\text { marcogczz@ outlook.com }}^{3}$ pereira.asp@gmail.com ${ }^{4} \underline{\text { luisfranz@gmail.com }}$ $5^{*}$ reheidtmann@yahoo.com.br}

\section{ARTIGO INFO.}

Recebido em: 08.11.2021

Aprovado em: 07.12.2021

Disponibilizado em: 17.12 .2021

Palavras-chave:

Dispositivos vestíveis; Robôs colaborativos; Methodi Ordinatio; Ergonomia.

KEYWORDS:

Wearable devices; Collaborative robots; Methodi Ordinatio; Ergonomics.

*Autor Correspondente: Heidtmann-Bemvenuti, R.

\section{RESUMO}

Segundo dados do Observatório Digital de Saúde e Segurança do Trabalho, desde 2012, o Brasil registrou mais de 17 mil mortes e 4,5 milhões acidentes de trabalho, sendo os afastamentos acidentários por acidentes mais frequentes (65\%) que por doenças (35\%). Entendendo o grande desafio que isso representa para a produtividade, economia e qualidade dentro das corporações, existem abordagens usando as tecnologias da indústria 4.0 para a prevenção de acidentes de trabalho. O objetivo deste trabalho foi realizar uma revisão sistemática sobre a utilização das tecnologias da Indústria 4.0 aplicadas à Saúde e Segurança do Trabalho para prevenção de acidentes de trabalho. Realizou-se uma revisão sistemática da literatura utilizando o Methodi Ordinatio, com a inclusão de artigos científicos publicados nas bases de dados Science Direct, Scopus e Web of Science. Foram utilizados termos de busca: "Industry 4.0"; "Occupational Health"; e "Safety"; utilizando o operador booleano AND. Foram encontrados 99 documentos, restando 94 após eliminação de artigos duplicados e 19 foram selecionados para análise e leitura minuciosa. Foram consideradas promissoras as possibilidades de uma gestão ocupacional preventiva e simultânea através do uso das tecnologias da indústria 4.0. A literatura reflete a importância de adaptações tecnológicas em termos de gestão da Saúde e Segurança do Trabalho no Brasil, de forma a torná-la mais propositiva, prática, simultânea e eficiente.

\begin{abstract}
According to data from the Digital Occupational Health and Safety Observatory, since 2012, Brazil has recorded more than 17 thousand deaths and 4.5 million work accidents, with accidental leaves being more frequent (65\%) than due to illness (35\%). Understanding the great challenge that this represents for productivity, economy and quality within corporations, there are approaches using industry 4.0 technologies for the prevention of work accidents. The objective of this work was to carry out a systematic review on the use of Industry 4.0 technologies applied to Occupational Health and Safety for the prevention of work accidents. A systematic literature review was carried out using the Methodi Ordinatio, with the inclusion of scientific articles published in databases Science Direct, Scopus and Web of Science. Search terms were used: "Industry 4.0"; "Occupational Health"; and "Safety"; using the Boolean AND operator. 99 documents were found, remaining 94 after the elimination of duplicate articles and 19 were selected for analysis and thorough reading. Were considered promising the possibilities of preventive and simultaneous occupational management using industry 4.0 technologies. Literature reflects the importance of technological adaptations in terms of Occupational Health and Safety management in Brazil, to make it more propositional, practical, simultaneous, and efficient.
\end{abstract}




\section{INTRODUÇÃO}

Ao longo da história, alguns estágios foram considerados grandes revoluções industriais, pois trouxeram à sociedade e à economia de cada época novos olhares e tendências sobre o uso de recursos, tecnologias e principalmente, sobre as relações de trabalho e suas perspectivas quanto à saúde e segurança do trabalhador (Sumer, 2018; Tessarini \& Saltorato, 2018; Adem, Çakit, \& Dagdeviren, 2020).

Há alguns anos, percebe-se uma mudança de paradigma na forma como o trabalho e a comunicação são realizados, denominada Quarta Revolução Industrial, que se caracteriza pelo uso de processos que utilizam máquinas gerenciadas por inteligência tecnológica (Pauliková, Babel'ová, \& Ubárová, 2021). O termo Indústria 4.0 (I4.0) tem sido recorrente para nomear a referida revolução industrial emergente nos anos recentes.

As inovações trazidas pelos conceitos da Indústria 4.0, que se baseiam na combinação de tecnologias aplicadas ao processo produtivo têm como princípios básicos os conceitos de Cyber-Physical Systems (CPS), Internet of Things (IoT), Internet of Services (IoS), robótica avançada, inteligência artificial, Big Data, nanomateriais e nanosensores (Neiva, Menezes, Lira, \& Brasileiro, 2020; Turk, Resman, \& Herakovic, 2020; Dhalmahapatra, Maiti, \& Krishna, 2021; Pauliková, Babel'ová, \& Ubárová, 2021; Petz, Eibensteiner, \& Langer, 2021).

Observa-se que junto com as evoluções das inovações tecnológicas que marcaram as revoluções industriais, houve um desenvolvimento da Saúde e Segurança do Trabalho (SST), e que as mudanças nos processos e métodos de produção ajudaram na implementação de soluções para os problemas de riscos ocupacionais (Badri, Boudreau-Trudel, \& Souissi, 2018).

Pontarolo (2018) verificou que, com a inserção de sistemas de SST, obteve-se redução de acidentes de trabalho e que o ensinamento do tópico aos colaboradores gera um espaço para que se sintam integrados e preocupados, ajudando nas definições de ações. Percebe-se, portanto, que pesquisas que buscam compreender como as tecnologias da I4.0 contribuem no contexto da Saúde e Segurança do Trabalhador emergem como uma importante lacuna de pesquisa (Badri et al., 2018).

Dessa forma, o presente trabalho apresenta um levantamento de tecnologias da I4.0 que vêm sendo aplicadas com foco na prevenção de acidentes de trabalho, abordagem essa ainda pouco explorada no Brasil.

\section{METODOLOGIA}

O presente trabalho trata-se de uma pesquisa exploratória, qualitativa de natureza básica. Foi realizado um estudo de Revisão Sistemática da Literatura (RSL), utilizando o protocolo Methodi Ordinatio sobre o uso das tecnologias da Indústria 4.0 na Segurança e Saúde do Trabalho.

A metodologia de RSL pondera três variáveis fundamentais para identificar a relevância das pesquisas de uma carteira bibliográfica: o fator de impacto da revista, o número de citações e o ano de publicação do artigo. Além disso, ela foi aplicada em mais de 200 pesquisas de revisão 
sistemática em diferentes áreas do conhecimento (Peixoto, Pereira-filho, \& Farias, 2021; Rolim, Gabriel, Mota, \& Quental, 2021).

Ao aplicar a equação InOrdinatio, os pesquisadores identificam, dentre os trabalhos selecionados, os mais relevantes para serem usados na revisão bibliográfica (Pagani, Kovaleski, \& Resende, 2015; Pagani, Kovaleski, \& Resende, 2018).

Este trabalho seguiu as nove etapas do Methodi Ordinatio (Figura 1) para classificar os trabalhos da produção acadêmica (Pagani et al., 2018).

Figura 1. Fluxograma das etapas da metodologia InOrdinatio

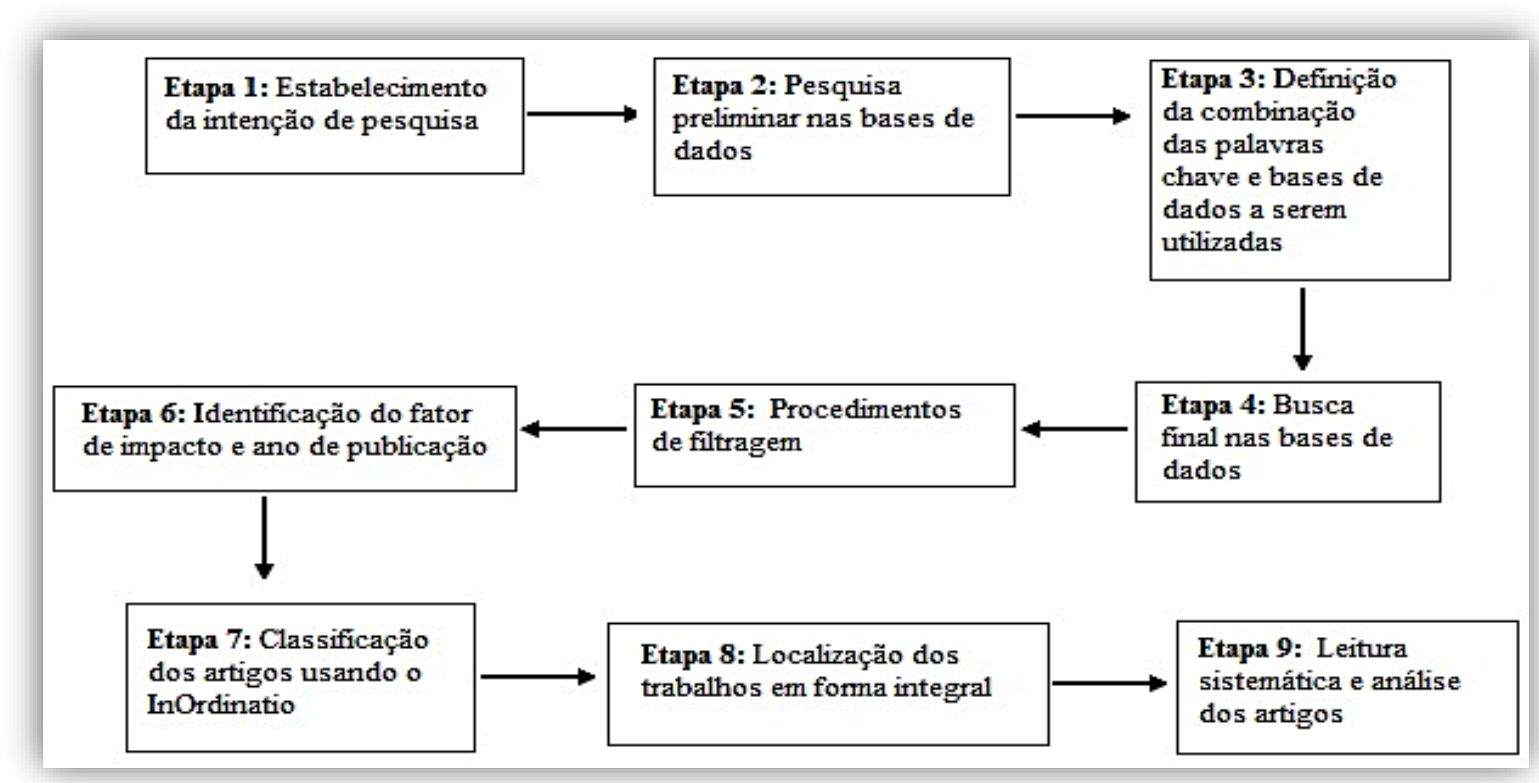

Fonte: Autores

A seguir, o Quadro 1 mostra as etapas um, dois, três e quatro da metodologia InOrdinatio.

Quadro 1. Etapas um a quatro do Methodi Ordinatio

\begin{tabular}{|c|c|}
\hline Etapa & Descrição \\
\hline 1 & Estabelecimento da intenção de pesquisa: Uso das tecnologias da Indústria 4,0 aplicadas à saúde e \\
segurança do trabalho
\end{tabular}

\section{Fonte: Autores}

As buscas preliminares nas bases de dados foram realizadas em setembro de 2021 utilizando algumas sequências de palavras-chave e operadores booleanos. Alguns termos eram muito amplos e retornavam muitos artigos, enquanto alguns termos específicos retornavam poucos artigos.

Na terceira etapa, realizou-se a definição da combinação das palavras-chave e das bases de dados a serem utilizadas. Foram selecionadas bases de dados consagradas em Engenharias III,

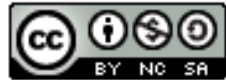


Citação (APA): Silva, C. M., da, Silva, M. A. G., da, Pereira, A. S., Franz, L. A. dos S., \& Heidtmann-Bemvenuti, R. (2021). Utilização das tecnologias da indústria 4.0 na segurança e saúde do trabalhador: uma revisão sistemática da literatura. Brazilian Journal of Production Engineering, 7(5), 252-268.

a qual engloba a Engenharia de Produção: Science Direct, Scopus, Web of Science. Além disso, essas bases retornaram maiores volumes de publicação com as palavras-chave utilizadas e maior disponibilidade de acesso ao material nelas publicado.

A opção "somente artigos" foi selecionada tendo em vista à exigência de se ter somente estudos primários em Revisões Sistemáticas da Literatura (Galvão \& Pereira, 2014).

Optou-se por não realizar limitação temporal nas buscas, para maior representatividade dos resultados obtidos.

$\mathrm{Na}$ etapa cinco, denominada procedimentos de filtragem, foram eliminados os artigos duplicados e, por meio da leitura dos títulos, foram eliminados aqueles não relacionados ao tema. Nesta etapa também foram eliminados artigos secundários e outros que não possuíam fator de impacto, como capítulos de livros e trabalhos de congressos, os quais a filtragem "somente artigos" não eliminou.

Estes procedimentos de filtragem resultaram em um novo número de trabalhos, que passou de 99 para 19 e que, posteriormente às etapas seis, sete e oito, passou para 14 artigos analisados (Tabela 1).

Tabela 1. Resultado Methodi Ordinatio com filtragem

\begin{tabular}{lc} 
Processo de Filtragem & Resultado \\
\hline Total de artigos & \\
Science Direct: 82 & 99 \\
Scopus: 7 (sete) & \\
Web of Science: 10 & 94 \\
s eliminação de artigos duplicados & 19 \\
não relacionados ao tema proposto ou sem fator de impacto & 14 \\
\hline igos analisados na RSL (etapa nove)
\end{tabular}

Fonte: Autores

A identificação do fator de impacto, ano de publicação e número de citações, prevista na etapa seis, foi feita utilizando-se os sites das revistas ou o Google Scholar. Dos 19 artigos, 17 utilizavam a métrica Scientific Journal Rankings (SJR) e dois a Journal Citation Reports (JCR).

Os dois grupos foram tratados separadamente na etapa sete e depois incorporados na mesma Tabela (Tabela 2), tendo em vista que os resultados não apresentaram incompatibilidade. Os artigos foram organizados com as seguintes colunas: título do artigo, ano de publicação, fator de impacto (SJR ou JCR) e número de citações.

$\mathrm{Na}$ etapa sete, que trata da classificação dos artigos usando Equação InOrdinatio, realizou-se o cálculo apresentado na Equação 1. Para estabelecimento do ponto de corte, para a leitura integral dos artigos, aplicou-se a regra de Pareto (80/20).

Em que:

$$
\text { InOrdinatio }=\left(\frac{F i}{1000}\right)+\alpha \times[10-(\text { Aat }- \text { Aar })]+\sum C i
$$

$F i$ = fator de impacto da revista (SJR ou JCR) em que o artigo foi publicado;

$\alpha=$ fator de ponderação, relacionado com o peso do ano de publicação, que varia de 1 a 10 . É atribuído pelo pesquisador, conforme a atualidade do tema. Neste trabalho, utilizou-se $\alpha=10$, pois Pagani et al. (2015) sugerem utilizar este valor quando a atualidade dos artigos analisados é um fator importante para pesquisa. 
Citação (APA): Silva, C. M., da, Silva, M. A. G., da, Pereira, A. S., Franz, L. A. dos S., \& Heidtmann-Bemvenuti, R. (2021). Utilização das tecnologias da indústria 4.0 na segurança e saúde do trabalhador: uma revisão sistemática da literatura. Brazilian Journal of Production Engineering, 7(5), 252-268

Aat $=$ ano da execução das buscas desta pesquisa (constante igual a 2021);

Aar = ano de publicação de cada artigo encontrado;

$\Sigma C i=$ somatório de citações do artigo.

A localização dos trabalhos em formato integral (etapa oito), foi realizada simultaneamente com a etapa seis, conforme orientam Pagani et al. (2018), por se tratar da visualização de forma integral dos artigos localizados.

Para leitura sistemática e análise dos artigos (etapa nove), o pesquisador atribui valores e critérios pessoais a fim de determinar quantos artigos irá ler, tendo em vista que os trabalhos se encontram, nesta fase, classificados por ordem de relevância (Pagani et al., 2018).

Foi aplicada a regra de Pareto (80/20) nos valores InOrdinatio, que estabeleceu os 14 primeiros artigos como ponto de corte para leitura integral.

\section{RESULTADOS E DISCUSSÃO}

A Tabela 2 apresenta os 19 artigos selecionados na etapa cinco bem como o número de citações, fator de impacto $(\mathrm{Fi})$ e o resultado do cálculo InOrdinatio de cada um deles.

Tabela 2. Resultado da equação InOrdinatio para os 19 artigos

\begin{tabular}{|c|c|c|c|}
\hline $\begin{array}{c}\text { Artigo } \\
\left(5^{\mathrm{a}} \text { etapa }\right) \\
\end{array}$ & $\begin{array}{l}\text { Citações } \\
\left(6^{\mathbf{a}} \text { etapa }\right)\end{array}$ & $\begin{array}{c}\text { Fi } \\
\left(6^{\mathbf{a}} \text { etapa }\right)\end{array}$ & $\begin{array}{c}\text { InOrdinatio } \\
\left(7^{\mathrm{a}} \text { etapa) }\right.\end{array}$ \\
\hline $\begin{array}{l}\text { 1. Teleoperated mobile robot with two arms: the influence of a human- } \\
\text { machine interface, VR training and operator age } \\
\text { (Grabowski, Jankowski, \& Wodzynski, 2021) }\end{array}$ & 8,0 & 3,63 & 108,04 \\
\hline $\begin{array}{l}\text { 2. Assessment of virtual reality based safety training simulator for electric } \\
\text { overhead crane operations } \\
\text { (Dhalmahapatra, Maiti, \& Krishna, 2021) }\end{array}$ & 7,8 & 4,88 & 107,85 \\
\hline $\begin{array}{l}\text { 3. Design and implementation of a computerized safety inspection system for } \\
\text { construction sites using UAS and digital checklists-Smart Inspecs } \\
\text { (Rey, Melo, \& Costa, 2021) }\end{array}$ & 7,8 & 4,88 & 107,85 \\
\hline $\begin{array}{l}\text { 4. Modern Cause and Effect Model by Factors of Root Cause for Accident } \\
\text { Prevention in Small to Medium Sized Enterprises } \\
\text { (Kang, Yang, \& Patterson, 2021) }\end{array}$ & 4,5 & 2,71 & 104,53 \\
\hline $\begin{array}{l}\text { 5. Sensor Shirt as Universal Platform for Real-Time Monitoring of Posture } \\
\text { and Movements for Occupational Health and Ergonomics } \\
\text { (Petz, Eibensteiner, \& Langer, 2021) }\end{array}$ & 3,0 & 2,40 & 103,02 \\
\hline $\begin{array}{l}\text { 6. Analysis of the Impact of Human-Cobot Collaborative Manufacturing } \\
\text { Implementation on the Occupational Health and Safety and the Quality } \\
\text { Requirements } \\
\text { (Pauliková, Babel'ová, \& Ubárová, 2021) }\end{array}$ & 2,4 & 3,40 & 102,44 \\
\hline $\begin{array}{l}\text { 7. Ergonomic risk assessment based on computer vision and machine learning } \\
\text { (Massiris Fernández, Fernández, Bajo \& Delrieux, 2020) }\end{array}$ & 7,9 & 5,43 & 97,95 \\
\hline $\begin{array}{l}\text { 8. Expected impact of industry } 4.0 \text { technologies on sustainable development: } \\
\text { A study in the context of Brazil's plastic industry } \\
\text { (Nara, Costa, Baierle, Schaefer, Benitez, Santos, \& Benitez, 2020) }\end{array}$ & 6,7 & 5,03 & 96,75 \\
\hline $\begin{array}{l}\text { 9. Design of human-centered collaborative assembly workstations for the } \\
\text { improvement of operators' physical ergonomics and production efficiency: A } \\
\text { case study } \\
\text { (Gualtieri, Palomba, Merati, Rauch, \& Vidoni, 2020a) }\end{array}$ & 3,9 & 3,25 & 93,93 \\
\hline $\begin{array}{l}\text { 10. From Design for Assembly to Design for Collaborative Assembly - } \\
\text { Product Design Principles for Enhancing Safety, Ergonomics and Efficiency } \\
\text { in Human-Robot Collaboration } \\
\text { (Gualtieri Rauch, Vidoni, \& Matt, 2020b) }\end{array}$ & 3,3 & 2,40 & 93,32 \\
\hline $\begin{array}{l}\text { 11. The impact of smart technologies: A case study on the efficiency of the } \\
\text { manual assembly process } \\
\text { (Turk, Resman, \& Herakovic, 2020) }\end{array}$ & 3,3 & 2,40 & 93,32 \\
\hline $\begin{array}{l}\text { 12. Holistic planning and optimization of human-centred workplaces with } \\
\text { integrated Exoskeleton technology } \\
\text { (Ippolito, Constantinescu, \& Riedel, 2020) }\end{array}$ & 3,3 & 2,40 & 93,32 \\
\hline
\end{tabular}

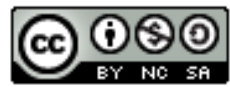


Citação (APA): Silva, C. M., da, Silva, M. A. G., da, Pereira, A. S., Franz, L. A. dos S., \& Heidtmann-Bemvenuti, R. (2021). Utilização das tecnologias da indústria 4.0 na segurança e saúde do trabalhador: uma revisão sistemática da literatura. Brazilian Journal of Production Engineering, 7(5), 252-268.

13. Ergonomic Design for Sausage Packing Hand Tool (Lohasiriwat \& Chaiwong, 2020)

14. Occupational health and safety risk assessment in the domain of Industry 4.0

(Adem, Çakit, \& Dagdeviren, 2020)

15. SafE-Tag mobile: A novel javascript framework for real-time management of unsafe conditions and unsafe acts in SMEs

(Vukicevic, Djapan, Stefanovic, \& Macuzic, 2019)

16. How to improve worker's well-being and company performance: a method to identify effective corrective actions (Scafa, Papetti, Brunzini, \& Germani, 2019)

17. Occupational health and safety in the industry 4.0 era: A cause for major concern?

(Badri, Boudreau-Trudel, \& Souissi, 2018)

18. Improving a production site from a social point of view: an IoT infrastructure to monitor workers condition

(Gregori, Papetti, Pandolfi, Peruzzini, \& Germani 2018)

19. Methodology for Monitoring Manufacturing Environment by Using

Wireless Sensor Networks (WSN) and the Internet of Things (IoT) (Li \& Kara, 2017)

$\begin{array}{lll}3,3 & 2,40 & 93,32 \\ 7,8 & 4,88 & 90,00 \\ 7,8 & 4,88 & 87,85 \\ 3,3 & 2,40 & 83,32 \\ 7,8 & 4,88 & 77,85 \\ 3,3 & 2,40 & 73,32 \\ 3,3 & 2,40 & 63,32\end{array}$

Fonte: Autores

A aplicação da regra de Pareto (80/20) nos valores InOrdinatio estabeleceu os 14 primeiros artigos como ponto de corte para leitura integral.

\section{Local das pesquisas}

É possível observar que todos os 14 artigos, selecionados na filtragem, referem-se a publicações de diversos locais ao redor do mundo (Quadro 2).

Quadro 2. Local das pesquisas de tecnologias da I4.0 na SST

\begin{tabular}{|c|c|}
\hline Local da pesquisa & Referência \\
\hline Alemanha & Ippolito et al. (2020) \\
\hline Argentina & Massíris Fernández et al. (2020) \\
\hline Áustria & Petz et al. (2021) \\
\hline \multirow{2}{*}{ Brasil } & Rey et al. (2021) \\
\hline Coreia & Nara et al. (2020) \\
\hline Eslováquia & Kang et al. (2021) \\
\hline Eslovênia & Pauliková et al (2021) \\
\hline Índia & Turk et al. (2020) \\
\hline \multirow{2}{*}{ Itália } & Dhalmahapatra et al. (2021) \\
\hline Polônia & Gualtieri et al. (2020a) \\
\hline Tailândia & Gualtieri et al. (2020b) \\
\hline Turquia & Grabowski et al. (2021) \\
\hline
\end{tabular}

Fonte: Autores

Esses resultados demonstram que as tecnologias da I4.0 vêm cada vez mais sendo utilizada em todo o mundo e é possível observar que, apesar de não ter sido restringido o ano nas buscas, os estudos são todos dos anos de 2020 e 2021, ou seja, esse assunto é iminente e, pode-se dizer, promissor para a segurança dos trabalhadores.

\section{Análise sistemática conforme temáticas dos artigos}

As formas de prevenção de acidentes de trabalho trazidas pelos 14 artigos selecionados foram organizadas conforme as principais tecnologias envolvidas na $4^{a}$ Revolução Industrial: Internet das Coisas (IoT), Machine Learning, Sistemas Ciber-Físicos (CPS) e Inteligência Artificial (IA)

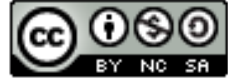


Citação (APA): Silva, C. M., da, Silva, M. A. G., da, Pereira, A. S., Franz, L. A. dos S., \& Heidtmann-Bemvenuti, R. (2021). Utilização das tecnologias da indústria 4.0 na segurança e saúde do trabalhador: uma revisão sistemática da literatura. Brazilian Journal of Production Engineering, 7(5), 252-268

(Filho 2015; Teixeira, Teixeira, Brito, \& Silva, 2019). Os resultados foram abordados conforme os seguintes tópicos: dispositivos vestíveis, robôs colaborativos, recursos tecnológicos aplicados à prevenção de acidentes e limitações associadas ao uso das tecnologias 4.0 (Quadro $3)$.

Quadro 3. Resumo das tecnologias da I4.0 que foram utilizadas por cada um dos 14 artigos analisados

\begin{tabular}{|c|c|c|}
\hline Tópicos & Tecnologias I4.0 & Artigo \\
\hline Dispositivos vestíveis & $\begin{array}{c}\text { IoT } \\
\text { Machine Learning } \\
\text { CPS }\end{array}$ & $\begin{array}{l}\text { Ippolito et al. (2020) } \\
\text { Petz et al. (2021) }\end{array}$ \\
\hline Robôs colaborativos & $\begin{array}{c}\text { IoT } \\
\text { Inteligência Artificial }\end{array}$ & $\begin{array}{l}\text { Gualtieri et al. (2020a) } \\
\text { Gualtieri et al. }(2020 \mathrm{~b}) \\
\text { Grabowski et al. }(2021) \\
\text { Pauliková et al. }(2021)\end{array}$ \\
\hline $\begin{array}{l}\text { Recursos tecnológicos aplicados } \\
\text { na prevenção de lesões e } \\
\text { acidentes }\end{array}$ & $\begin{array}{c}\text { Machine Learning } \\
\text { IoT } \\
\text { CPS } \\
\text { Inteligência Artificial }\end{array}$ & $\begin{array}{c}\text { Massíris et al. (2020) } \\
\text { Turk et al. }(2020) \\
\text { Dhalmahapatra et al. }(2021) \\
\text { Rey et al. }(2021)\end{array}$ \\
\hline $\begin{array}{c}\text { Limitações associadas ao uso das } \\
\text { tecnologias da I4.0 }\end{array}$ & ( & $\begin{array}{c}\text { Lohasiriwat e Chaiwong (2020) } \\
\text { Kang et al. }(2021) \\
\text { Nara } \text { et al. }(2020) \\
\text { Adem } \text { et al. }(2020)\end{array}$ \\
\hline
\end{tabular}

Fonte: Autores

A Internet das Coisas (ou Internet of Things - IoT) representa a possibilidade de os objetos físicos estarem conectados à internet, podendo dessa forma executar uma determinada ação (Teixeira et al., 2019). O aprendizado das máquinas (ou Machine Learning), é uma metodologia de análise de dados, que se utiliza de algoritmos que respondem e se adaptam automaticamente aos dados sem a necessidade de intervenção humana contínua (Filho, 2015). Os sistemas ciber físicos (ou Cyber-Physical Systems - CPS), por sua vez, visam sintetizar a fusão entre o mundo físico e digital. Dentro desse conceito, todo o objeto físico (seja uma máquina ou uma linha de produção) e os processos físicos que ocorrem, em função desse objeto, são digitalizados. A Inteligência Artificial (IA) é um segmento da computação que busca simular a capacidade humana de raciocinar, tomar decisões, resolver problemas, dotando softwares e robôs de uma capacidade de automatizarem vários processos (Teixeira et al., 2019).

Liu et al. (2020), explicam que as tecnologias da I4.0 e a gestão ocupacional devem estar alinhadas de forma a melhorar a segurança do trabalhador e do ambiente de trabalho, ao mesmo tempo em que melhora a eficiência e otimiza a produção.

\section{Dispositivos Vestíveis}

Pôde-se notar que alguns estudos contemplam o uso dos "dispositivos vestíveis", conhecidos pela terminologia de wearables. Estes dispositivos têm como propósito principal o monitoramento e proteção contra as desordens musculoesqueléticas (Ippolito et al., 2020; Petz et al., 2021).

No artigo de Petz et al. (2021) é desenvolvido uma camisa, também chamada de dispositivo vestível que monitora e registra, por meio de sensores, os movimentos e a posição da parte superior do corpo.

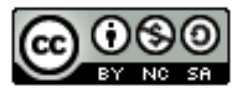


Por causa de suas propriedades flexíveis e sua proximidade natural com o corpo humano, os têxteis são uma camada protetora contra influências ambientais. O próximo passo no desenvolvimento de têxteis é a conexão com a eletrônica que, por meio de sensores, irá transmitir valores via Wi-Fi (Petz et al., 2021).

A camisa tem oito posições de sensor distribuídas, que estão na área do pescoço, ombros, braços e antebraços, peito, lombar e quadril. Este último também é usado para transmissão de dados, além de gravação de dados. Este nó é equipado com recursos de Wi-Fi e Bluetooth e fornece a fonte de alimentação para camisa. Os nós sensores são responsáveis por medir o movimento ou dados ambientais.

Foram testados movimentos simples, postura inclinado para trás, reto, inclinado para frente, movimentos mais complexos como de girar, pegar pacote da mesa, colocar pacote na mesa, tirar pacote da prateleira e colocar pacote na prateleira, com o objetivo de validar o projeto (Petz et al., 2021).

O uso de diferentes sensores permite uma adaptação rápida aos novos requisitos na medição e detecção de movimentos humanos. Apenas o número e a posição dos nós sensores devem ser definidos no início. Além disso, o design oferece a possibilidade de um microcontrolador designado em cada posição do sensor. Assim, a detecção de diferentes posições e movimentos pode ser feita na camisa e não requer qualquer transmissão a um computador host para processamento. Este sistema criado pode ser utilizado para registrar e classificar seções de movimento para análises ergonômicas, sequência de movimentos ou fluxos de trabalho (Petz et al., 2021).

No estudo de Ippolito et al. (2020) são levantados os benefícios dos exoesqueletos, a sua composição material, bem como as abordagens metodológicas nos quais os mesmos podem ser usados e implementados dentro das plantas fabris, gerando proteção e maior consideração de aspectos da Ergonomia nos ambientes produtivos.

Os autores fazem uma abordagem holística, sobre como integrar o exoesqueleto na área de produção por meio dos tipos de operações: análise e avaliação da ergonomia, dos produtos e das ferramentas de trabalho (feita por meio de análise de tempos e movimentos e usando o processo de simulação por meio de software apropriado capaz de criar acoplamento entre humano e exoesqueleto); avaliação dos tipos de exoesqueletos apropriados para os trabalhadores daquele ambiente - por meio de modelagem digital por métodos de Task Analysis e Toolkit usado para ergonomia (Ippolito et al., 2020).

Por meio do uso do exoesqueleto na área de produção é possível ainda analisar as partes do corpo que estão mais suscetíveis a doenças musculoesqueléticas e selecionar o melhor exoesqueleto para o ambiente de trabalho, gerando uma personalização para diferentes tipos de ambientes e setores (Ippolito et al., 2020).

Com essas possibilidades, os profissionais de ergonomia podem acompanhar e monitorar os dados a fim de atuar com foco na minimização de doenças e acidentes ocupacionais. 
Citação (APA): Silva, C. M., da, Silva, M. A. G., da, Pereira, A. S., Franz, L. A. dos S., \& Heidtmann-Bemvenuti, R. (2021). Utilização das tecnologias da indústria 4.0 na segurança e saúde do trabalhador: uma revisão sistemática da literatura. Brazilian Journal of Production Engineering, 7(5), 252-268

A possibilidade de um ambiente integrado e digitalizado, onde as coisas se interconectam gerando uma gestão funcional, ocupacional e de segurança alinhadas é o propósito das mudanças conhecidas como Indústria 4.0 (Aslan, 2019).

\section{Robôs Colaborativos}

As empresas de manufatura devem considerar o elemento humano como essencial e valioso, melhorando as condições de trabalho e desenvolvendo a produção centrada no ser humano. É neste contexto que emerge a importância e protagonismo da Ergonomia (Gualtieri et al., 2020a).

Tendo em vista a relação entre os aspectos físicos, cognitivos e organizacionais da interação entre homens e sistemas, dois trabalhos recuperados do Methodi Ordinatio tratam dos robôs colaborativos, seus requisitos, especificações, benefícios à SST e posto de trabalho: Gualtieri et al. (2020a) e Gualtieri et al. (2020b).

O objetivo da interação humano-robô é a combinação das forças da automação com as habilidades únicas do ser humano, criando um ambiente seguro que gera maior lucro em um ambiente de trabalho. A interação humana-robô nos postos de trabalho visa melhorar a ergonomia física para os operadores, por meio de redução da sobrecarga biomecânica, e melhorar a produtividade da empresa, em termos de tempo de ciclo (Gualtieri et al., 2020a).

Gualtieri et al. (2020b) também relatam que é necessária a obtenção da maximização do bemestar do operador durante a interação com o robô e com outros elementos da estação de trabalho em termos físicos e cognitivos, bem como obter a minimização dos tempos e os custos das tarefas manuais, robóticas e tarefas colaborativas.

Esses mesmos autores fizeram um estudo de caso aplicado que apresentou melhorias nos aspectos das funções manuais e posturais, além de trazer eficiência produtiva. $O$ principal motivador para a conversão para um sistema colaborativo é a melhoria física dos operadores.

Os autores propõem um modelo sugerindo a implementação segura com os passos a serem analisados de forma a minimizar riscos ocupacionais, tanto nas atividades repassadas aos robôs como na interação entre homem e máquina seja introduzida de maneira segura, não gerando outros tipos de riscos ocupacionais (Gualtieri et al., 2020b).

O artigo de Grabowski et al. (2021) trata da utilização de técnicas de realidade virtual para controlar um robô móvel que permite carregar uma carga de $20 \mathrm{~kg}$, sendo o preço de implementação baixo, podendo ser utilizado em massa na indústria. $\mathrm{O}$ estudo foi realizado com 81 participantes sendo 63 jovens e 18 trabalhadores mais experientes. Três fatores foram levados em consideração, como interface homem-máquina, treinamento em realidade virtual e operador (Grabowski et al., 2021).

A vantagem de se trabalhar com robôs colaborativos é que os funcionários não precisam ficar expostos em áreas perigosas, como por exemplo, locais com muita poeira, alta temperatura ou com risco de explosão (Grabowski et al., 2021).

O objetivo principal do estudo foi investigar a possibilidade de usar robôs teleoperados de custo baixo por jovens e trabalhadores mais experientes, pois a idade pode ser um fator importante na adoção de novas tecnologias no local de trabalho (Grabowski et al., 2021). 
O robô construído seria utilizado principalmente em trabalho físico relacionado a mover ou organizar objetos, preservando a saúde do trabalhador. Um teleoperador pode supervisionar um grupo de robôs e controlar um robô selecionado em qualquer situação incomum ou de emergência. Estes tipos de robôs controlados remotamente podem realizar tarefas relacionadas a proteger uma fábrica após um acidente ou remover seus efeitos, como por exemplo parar o vazamento de substâncias perigosas (Grabowski et al., 2021).

O artigo de Pauliková et al. (2021) busca analisar o impacto da utilização de robôs colaborativos na saúde e segurança do trabalho. A tarefa é criar cooperação e colaboração entre um robô e um humano em um local de trabalho robotizado comum para que seja seguro e eficaz. $\mathrm{O}$ objetivo da pesquisa apresentada foi identificar, além dos efeitos positivos, os efeitos negativos em humanos na interação humano-cobot, garantindo a saúde e segurança no trabalho.

Segundo Pauliková et al. (2021) a indústria 4.0 é a combinação de inúmeras tecnologias físicas e digitais, como robótica adaptativa, inteligência artificial, realidade aumentada, computação em nuvem e internet das coisas.

A robotização do local de trabalho traz inúmeros benefícios para os trabalhadores. Ao realizar o trabalho em ambientes com substâncias químicas perigosas que são cancerígenas e tóxicas para os humanos, o robô acaba preservando a saúde dos trabalhadores. Além disso, ajuda a melhorar a ergonomia do local de trabalho.

Os autores afirmam que colocando robôs em operações de alto risco e trabalhos com cargas, podem significativamente reduzir as doenças ocupacionais. Futuramente, locais de trabalho colaborativos serão cada vez mais introduzidos na indústria. Segurança e redução de riscos de acidentes continuarão sendo uma prioridade (Pauliková et al., 2021).

Por outro lado, a interação com robôs pode expor o funcionário a riscos de saúde e segurança relacionados a máquinas automatizadas associadas ao estresse psicossocial. Estudos de segurança mostram que muitos acidentes em um local de trabalho robotizado ocorrem durante situações operacionais não rotineiras, como programação, manutenção, teste, configuração ou personalização. Durante essas operações, o funcionário pode acessar temporariamente a área de trabalho do robô, onde operações não intencionais podem causar ferimentos em uma pessoa. Além disso, a falta de experiência de trabalho no sistema humano-robô, a falta de treinamento de funcionários e a eliminação de alguns postos de trabalho também são apontados como aspectos negativos advindos da utilização das tecnologias para a SST (Pauliková et al., 2021).

\section{Recursos tecnológicos aplicados à prevenção de acidentes}

No artigo de Dhalmahapatra et al. (2021), é feita a avaliação e eficácia de um sistema de segurança imersivo baseado em realidade virtual, desenvolvendo um simulador de treinamento que permitiu treinar os operadores de pontes rolantes elétricas, com o objetivo de ajudar os novatos a compreender a sequência de operações, além de gerenciar os riscos potenciais durante a realização do trabalho.

Esses autores propuseram este treinamento baseado em realidade virtual devido à sua capacidade de visualização em tempo real, dinâmica modelagem de causalidade de acidentes, interação homem-máquina sem risco e melhora na taxa de aprendizagem. A realidade virtual é 
um ambiente organizado por meio de simulação de computador que conecta a presença de um usuário entre real e ambiente artificial. O objetivo do experimento é descobrir a eficácia da realidade virtual comparado aos métodos de treinamento do mundo real.

A configuração experimental inclui Head Mounted Display (HMD), joystick profissional 3D e Unreal Engine ambiente virtual. O usuário usa óculos com display e sensores que ajudam na posição do usuário. O sistema de rastreamento fornece o meio ideal para movimentos precisos e suaves, permitindo uma interação realista e intuitiva entre o usuário e o conteúdo do aplicativo (Dhalmahapatra et al., 2021).

Foi verificado que não há diferença significativa entre os jovens e operadores experientes em treinamentos de realidade virtual. $\mathrm{O}$ simulador de treinamento de segurança baseado em realidade virtual foi considerado eficaz.

No artigo de Rey et al. (2021) foi feita uma análise do número de acidentes de trabalho no Brasil, sendo o setor da construção a segunda atividade que mais mata no país, responsável por 4\% das mortes. Em seu estudo, foi proposto a utilização de recursos da I4.0 para realizar inspeções de segurança nas construções. Smart Inspecs reduz o tempo de inspeção de segurança, além de oferecer melhorias na interação entre a equipe, segurança e correção mais rápida de não conformidades de segurança (Rey et al., 2021).

O estudo propõe a implementação de um protótipo de sistema de inspeção de segurança computadorizado chamado Smart Inspecs que usa um sistema aéreo não tripulado (UAS) e uma lista de verificação digital para realizar as inspeções de segurança na construção, ou seja, utiliza uma tecnologia de aquisição de dados para monitorar as condições de segurança no local de trabalho. Essas tecnologias combinadas fornecem uma avaliação mais rápida e precisa além de ter uma confiabilidade maior (Rey et al., 2021).

O protótipo do Smart Inspecs foi projetado a partir da definição de protocolos de inspeção, definição de tecnologia UAS e definição de ferramentas de checklist digital (Rey et al., 2021).

Os resultados mostram que o tempo necessário para os dados de processamento e entrega dos relatórios para a equipe de segurança foi reduzido em $73 \%$ ao utilizar a abordagem proposta. $\mathrm{O}$ artigo mostrou que o uso das tecnologias existentes pode minimizar erros, evitando acidentes de trabalho.

No artigo de Massíris et al. (2020) é feito o acompanhamento e monitoramento de métodos ergonômicos com a ajuda das tecnologias da I4.0.

Várias tecnologias disruptivas relacionadas à I4.0 estão permitindo ferramentas e modelos proveitosos para o estudo e avaliação ergonômica.

Massíris et al. (2020) propuseram uma metodologia baseada em visão por computador capaz de fornecer avaliação ergonômica consistente e robusta, superando as limitações das abordagens atuais.

Foram realizados estudos com grupos de pessoas em tarefas de trabalho diversas, mas que tinham em comum o movimento repetitivo de membros superiores. Foi analisada a interação entre homem e trabalho a fim de detectar os movimentos repetitivos, força aplicada e amplitude do movimento angular para as profissões de flanelinha de aviões, servente de obra e bombeiro. 
Citação (APA): Silva, C. M., da, Silva, M. A. G., da, Pereira, A. S., Franz, L. A. dos S., \& Heidtmann-Bemvenuti, R. (2021). Utilização das tecnologias da indústria 4.0 na segurança e saúde do trabalhador: uma revisão sistemática da literatura. Brazilian Journal of Production Engineering, 7(5), 252-268

Para cada uma dessas profissões, os autores analisaram duas tarefas de trabalho onde existia uma grande frequência de repetição de movimentos dos membros superiores (Massíris et al., 2020).

Para avaliação das tarefas de trabalho, foi usado um software de código aberto com aprendizado de máquina (princípio do Machine Learning) que permite a leitura e detecção de articulações e membros a partir de imagens e vídeos digitais em tempo real. São usadas e programadas possíveis posturas para essas atividades. Após recolhimento das posturas pelas filmagens, é feito uma abordagem da movimentação do corpo e é criado um modelo vetorial das posições apresentadas, comparando com as armazenadas, a fim de verificar as diferenças entre os ângulos dos movimentos e garantir confiabilidade ao modelo (Massíris et al., 2020).

Esse é um método simples de levantamento de informações com fins na investigação ergonômica nos postos de trabalho que possuam potencial causador de desordens musculoesquelético muito usado para esse tipo de análise ergonômica (Massíris et al., 2020).

Conforme Massíris et al. (2020), a utilização das tecnologias da I4.0 pode auxiliar, facilitar e otimizar a gestão e elaboração de relatórios ergonômicos existentes e dar maior precisão para a conclusão de riscos de doenças ocupacionais.

No artigo de Turk et al. (2020), é apresentada uma nova estação de montagem manual equipada com tecnologias e ferramentas inteligentes. A estação de trabalho manual é autoconfigurável de acordo com o trabalhador, variedade do produto e estrutura do produto. Esta nova estação tem como objetivo otimizar o processo de montagem e minimizar os tempos de montagem, proporcionando condições ergonômicas de trabalho.

O estudo foi dividido em três partes, sendo a primeira a apresentação do local de trabalho e as diferenças entre as estações de montagem manual tradicional e inteligente. A segunda parte lida com o experimento de comparação entre as duas estações de trabalho e a terceira etapa avalia a ergonomia (Turk et al., 2020).

Foram selecionados dois produtos diferentes para o experimento, sendo realizada a montagem por empregados na estação de trabalho tradicional e na mais inteligente. Para avaliação da ergonomia foi utilizado o Siemens Jack DHM, software no qual foi importado os modelos CAD de ambas as estações (Turk et al., 2020).

A análise de erros mostrou que o uso de tecnologia inteligente reduz o número de erros em três vezes. Na análise ergonômica foi demonstrado que o processo de montagem do primeiro produto na estação de trabalho tradicional força o trabalhador a alcançar peças na zona perigosa, duas vezes mais que em uma estação de trabalho de montagem inteligente. As análises mostraram que a estação de montagem inteligente é recomendada para a saúde (análise ergonômica) bem como para produtividade (tempo e erros) (Turk et al., 2020).

\section{Limitações associadas ao uso das tecnologias da I4.0}

As chamadas tecnologias disruptivas referem-se a inovações tecnológicas e vêm se popularizando na área de SST em prol da prevenção de acidentes e doenças ocupacionais. $\mathrm{O}$ objetivo principal da utilização dessas tecnologias da Indústria 4.0 é atingir as necessidades do mercado de forma mais rápida e eficiente. No entanto, quando se trata da segurança e saúde do 
trabalhador, surgem alguns questionamentos sobre as aplicações e modificações trazidas por essas tecnologias (Badri et al., 2018; Liu et al., 2020).

Lohasiriwat e Chaiwong (2020) refletem sobre os danos que a prevalência do risco ergonômico pode causar aos trabalhadores em uma empresa de fabricação de salsichas. Foi investigado o risco a saúde do trabalhador no setor que apresenta continuamente maiores incidências de lesões, sendo ele o setor de embalagem. Estes riscos ocorrem devido ao novo ambiente de trabalho em fábricas inteligentes, no qual o homem tem que acompanhar a velocidade das máquinas.

Realizaram-se testes por meio do redesenho de trabalho, no qual favoreceria a ergonomia do punho, reduzindo os danos causados pelo trabalho. Além disso, protótipos foram criados com o intuito de auxiliar na execução das tarefas.

Kang et al. (2021) analisam os efeitos causadores de acidente em empresas de pequeno e médio porte na Coreia do Sul. Para esta avaliação foi utilizado o modelo moderno de causa e efeito que inclui fatores de causa raiz.

Para minimizar os acidentes de trabalho, é importante priorizar fatores e estratégias de prevenção que sistematicamente fornecem informações para estabelecer um método útil de prevenção de acidentes e gerenciamento de segurança. A criação do modelo moderno de causa e efeito, prioriza fatores de causa raiz (Kang et al., 2021).

Os acidentes por fatores de causa raiz em sistemas robóticos e sistemas inteligentes como a Indústria 4.0 têm mais complexidade do que os sistemas atuais. Assim, esses sistemas devem realizar análises de intensidade a fim de minimizar os acidentes de trabalho fatais. Os resultados permitem propor políticas educacionais que minimizem os acidentes mais comuns pela causa (Kang et al., 2021).

Esses autores desenvolveram o modelo moderno de causa e efeito a fim de minimizar os acidentes de trabalho e acidentes fatais por meio de análise de intensidade. Foi proposto um sistema de educação em saúde ocupacional, com o objetivo de conscientizar sobre a saúde e segurança, prevenindo acidentes de ocorrência comum e um sistema de bloqueio e etiquetagem em equipamentos, para prevenir acidentes causados pelo uso inadequado de dispositivos de proteção.

Nara et al. (2020) analisaram o impacto das tecnologias da indústria 4.0 na sustentabilidade. Foi utilizado a perspectiva do Tripé da Sustentabilidade. Este modelo foi testado na indústria de plásticos que possui alto potencial tecnológico da indústria 4.0. Dentre os resultados obtidos, IoT, sensores e CPS tem uma influência positiva na eficiência energética, como consumo de água e matéria prima. Isso pode ser explicado pela sistematização e controle de tarefas nos processos produtivos da indústria do plástico proporcionado por essas tecnologias. Consequentemente, isso leva a redução de desperdício de energia, água e outros fatores (Nara et al., 2020).

Os resultados indicaram também que a implementação de tecnologias da indústria 4.0 tem impacto positivo no pilar ambiental. Integrando a IoT, sensores e big data na indústria de plásticos, é possível reduzir o uso de recursos e aumentar o uso de recursos renováveis e 
recicláveis. Além disso, a IoT e os sensores também reduzem a emissão de poluentes e efluentes com o uso eficiente de recursos na área produtiva (Nara et al., 2020).

Os autores consideram que essas tecnologias têm um impacto positivo na saúde e segurança do trabalho. Isso ocorre porque o monitoramento e controle das operações fornecidas por essas tecnologias evitam situações perigosas e não expõem os trabalhadores a riscos desnecessários (Nara et al., 2020).

Adem et al. (2020) refletem sobre a necessidade de considerar os possíveis riscos em termos de SST provenientes do novo ambiente de trabalho das fábricas inteligentes (Smart Factories).

Alguns fatores de potencial risco dentro da nova realidade são desordens relativas à visão ou problemas nos olhos (principalmente inerentes a interações com telas e dispositivos digitais de leitura e análise); fadiga mental; distúrbios decorrentes de posições estáticas de trabalho; exposição a agentes desconhecidos (perigosos) devido a cooperação com robôs; e pressão psicológica derivada de problemas de adaptação às funções que requerem criatividade e uso de tecnologias digitais (Adem et al., 2020).

Nesse sentido, Goelzer, (2020) alerta que, independente das modificações no mundo do trabalho, com novas tecnologias e novos cenários que exigem adaptações na prática da SST, a antecipação, reconhecimento, avaliação, prevenção e controle dos riscos não podem mudar. A mesma autora comenta que entre 2007 e 2017 houve aumento de quatro vezes no número de robôs na indústria e que pelo menos $46 \%$ dos trabalhos existentes hoje serão modificados pela automação.

Apesar de os artigos analisados nesta RSL abordarem predominantemente pontos positivos da implantação das tecnologias na SST, sabe-se que algumas consequências podem ser desencadeadas, como a redução da criatividade, devido à automação das máquinas, o desaparecimento do capital humano de produção e o aumento da taxa de desemprego (Sima et al., 2020).

\section{CONSIDERAÇÕES FINAIS}

A partir do presente estudo, foi possível uma revisão sistemática da literatura, utilizando o protocolo Methodi Ordinatio, sobre a utilização das tecnologias da Indústria 4.0 aplicadas à Saúde e Segurança do Trabalho para prevenção de acidentes de trabalho.

É possível considerar como promissora as possibilidades de uma gestão ocupacional preventiva e simultânea utilizando as tecnologias da indústria 4.0.

A literatura trazida como resultado deste trabalho reflete a importância de adaptações tecnológicas em termos de gestão da segurança e saúde do trabalhador no Brasil, tornando-a mais propositiva, prática, simultânea e eficiente.

Tecnologias como IoT, sensores e CPS mostraram impacto positivo na eficiência energética, consumo de água e de matéria prima, explicada pela sistematização e controle de tarefas nos processos produtivos da indústria que se utiliza dessas tecnologias.

O monitoramento e controle das operações utilizando essas tecnologias mostraram-se promissores na prevenção de situações perigosas. 
Citação (APA): Silva, C. M., da, Silva, M. A. G., da, Pereira, A. S., Franz, L. A. dos S., \& Heidtmann-Bemvenuti, R. (2021). Utilização das tecnologias da indústria 4.0 na segurança e saúde do trabalhador: uma revisão sistemática da literatura. Brazilian Journal of Production Engineering, 7(5), 252-268

Em contrapartida, também há aspectos preocupantes como o agravamento de doenças de cunho, principalmente, psicológicos que essas mudanças tecnológicas podem trazer no mundo do trabalho. Além disso, foi verificada a prevalência do risco ergonômico em fábricas inteligentes em que o homem tem que acompanhar a velocidade das máquinas.

As perspectivas quanto ao uso das tecnologias da Indústria 4.0 aplicadas à SST mostraram ter características de sincronicidade com o sistema e capacidade de tomada de decisão em tempo real por meio do uso de dados e características precisas, tendo um nível de assertividade que poderá reduzir os acidentes de trabalho e doenças ocupacionais.

Sugere-se, como trabalhos futuros, que seja realizado um levantamento da relação entre a satisfação dos funcionários, a qual tem relação direta com a produtividade, e a utilização das tecnologias da I4.0 na SST.

\section{REFERÊNCIAS}

Adem, A., Çakit, E., \& Dağdeviren, M. (2020). Occupational health and safety risk assessment in the domain of Industry 4.0. Applied Sciences, 2(977), 1-6. https://doi.org/10.1007/s42452$\underline{020-2817-x}$

Aslan, I. (2019). The Role of Industry 4.0 in Occupational Health and Safety. International European Congress On Social Sciences - IV, Diyarbakır, Turquia, p. 334-345. Recuperado de https://www.researchgate.net/publication/336699164

Badri, A., Boudreau-Trudel, B., \& Souissi, A. S. (2018). Occupational health and safety in the industry 4.0 era: A cause for major concern? Safety Science, 109, 403-411. https://doi.org/10.1016/j.ssci.2018.06.012

Dhalmahapatra, K., Maiti, J., \& Krishna, O. B. (2021). Assessment of virtual reality based safety training simulator for electric overhead crane operations. Safety Science. 139. https://doi.org/10.1016/j.ssci.2021.105241

Di Nardo, M., Madonna, M., Murino, T., \& Castagna, F. (2020). Modelling a Safety Management System Using System Dynamics at the Bhopal Incident. Applied Science, 10(3), 903. https://doi.org/10.3390/app10030903

Filho, A. D. P. C. (2015). Uso de big data em saúde no Brasil: perspectivas para um futuro próximo. Epidemiologia e Serviços de Saúde. 24(2), 325-332. Recuperado de http://scielo.iec.gov.br/pdf/ess/v24n2/v24n2a15.pdf

Galvão, T. F. \& Pereira, M. G. (2014). Revisões sistemáticas da literatura: passos para sua elaboração. Epidemiologia e Serviços de Saúde. 23(1), 183-184. Recuperado de http://scielo.iec.gov.br/pdf/ess/v23n1/v23n1a18.pdf

Goelzer, B. (2020, Setembro). Mudanças no mundo do trabalho e seus efeitos na SST. Revista Proteção, 11(347). Recuperado de https://protecao.com.br/eventos/mudancas-no-mundo-dotrabalho-e-seus-efeitos-na-sst-foi-tema-no-congresso-sst-2020/

Grabowski, A., Jankowski, J., \& Wodzynski, M. (2021). Teleoperated mobile robot with two arms: the influence of a human-machine interface, VR training and operator age. International Journal of Human - Computer Studies. 156. https://doi.org/10.1016/j.ijhcs.2021.102707

Gregori, F., Papetti, A., Pandolfi, M., Peruzzini, M., \& Germani, M. (2018). Improving a production site from a social point of view: an IoT infrastructure to monitor workers condition. Procedia CIRP, 72, 886-891. https://doi.org/10.1016/j.procir.2018.03.057 
Citação (APA): Silva, C. M., da, Silva, M. A. G., da, Pereira, A. S., Franz, L. A. dos S., \& Heidtmann-Bemvenuti, R. (2021). Utilização das tecnologias da indústria 4.0 na segurança e saúde do trabalhador: uma revisão sistemática da literatura. Brazilian Journal of Production Engineering, 7(5), 252-268

Gualtieri, L., Palomba, I., Merati, F. A., Rauch, E., \& Vidoni, R. (2020a). Design of HumanCentered Collaborative Assembly Workstations for the Improvement of Operators' Physical Ergonomics and Production Efficiency: A Case Study. Sustainability, 12(9). https://doi.org/10.3390/su12093606

Gualtieri, L., Rauch, E., Vidoni, R., \& Matt, D. T. (2020b) Safety, ergonomics and efficiency in Human-Robot Collaborative Assembly: Desing Guidelines and Requirements. Procedia CIRP, 91, 367-372. https://doi.org/10.1016/j.procir.2020.02.188

Ippolito, D., Constantinescu, C., \& Riedel, O. (2020) Holistic Planning and optimization of human-centred workplaces with integrated Exoskeleton technology. Procedia CIRP, 88, 214217. https://doi.org/10.1016/j.procir.2020.05.038

Kang, Y., Yang, S., \& Patterson, P. (2021). Modern Cause and Effect Model by Factors of Root Cause for Accident Prevention in Small to Medium Sized Enterprises. Safety and Health at Work. https://doi.org/10.1016/j.shaw.2021.08.002

Li, W. \& Kara, S. (2017). Methodology for Monitoring Manufacturing Environment by Using Wireless Sensor Networks (WSN) and the Internet of Things (IoT). Procedia CIRP, 61, 323328. https://doi.org/10.1016/j.procir.2016.11.182

Lohasiriwat, H. \& Chaiwong, W. (2020) Ergonomic Design for Sausage Packing Hand Tool. Procedia CIRP. 91, 789-795. https://doi.org/10.1016/j.procir.2020.02.236

Lo Presti, D., Carnevale, A., D’Abbraccio, J., Massari, L., Massaroni, C., Sabbadini, R., Zaltieri, M., Di Tocco, J., Bravi, M., Miccinilli, S., Sterzi, S., Longo, U. G., Denaro, V., Caponero, M. A., Formica, D., Oddo, C. M., \& Schena, E. (2020). A Multi-Parametric Wearable System to Monitor Neck Movements and Respiratory Frequency of Computer Workers. Sensors. 20(2), 536. https://doi.org/10.3390/s20020536

Massiris, F. M., Fernández, J. Á., Bajo, J. M., \& Delrieux, C. A. (2020). Ergonomic risk assessment based on computer vision and machine learning. Computers \& Industrial Engineering. https://doi.org/10.1016/j.cie.2020.106816

Nara, E. O. B., da Costa, M. B., Baierle, I. C., Schaefer, J. L., Benitez, G. B., do Santos, L. M. A. L., \& Benitez, L. B. (2020). Expected Impact of Industry 4.0 Technologies on Sustainable Development: A study in the context of Brazil's Plastic Industry. Sustainable Production and Consumption. https://doi.org/10.1016/j.spc.2020.07.018

Neiva, L. S., de Menezes, L. N., Lira, M. C. de A. \& Brasileiro, M. I. (2020). Industry 4.0: concepts, challenges and expectations. Revista Brasileira de Engenharia de Produção - BJPE. 6(1), 1-10. Recuperado de https://periodicos.ufes.br/bjpe/article/view/28247

Pagani, R. N., Kovaleski, J., \& Resende, L. M. (2015). Methodi Ordinatio: a proposed methodology to select and rank relevant scientific papers encompassing the impact factor, number of citations, and year of publication. Scientometrics, 105(3), 2109-2135. https://doi.org/10.1007/s11192-015-1744-x

Pagani, R., Kovaleski, J., \& Resende, L. M. (2018). Avanços na composição da Methodi Ordinatio para revisão sistemática de literatura. Ciência da Informação. 46(2), 161-187. Recuperado de http://revista.ibict.br/ciinf/article/download/1886/3708/12368

Pauliková, A., Babel'ová, Z. G., \& Ubárová, M. (2021). Analysis of the Impact of HumanCobot Collaborative Manufacturing Implementation on the Occupational Health and Safety and the Quality Requirements. International Journal of Environmental Research and Public Health, 18(4), 1927. https://doi.org/10.3390/ijerph18041927

Peixoto, N. E. S., Pereira-filho, M. M., \& Farias, J. S. (2021). Panorama da Produção Empírica Internacional de Inovação em Serviços Turísticos entre 2008 e 2020: Uma Revisão Sistemática

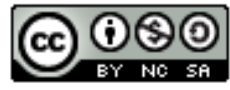

Esta obra está licenciada com uma Licença Creative Commons Atribuição-Não Comercial-Compartilha Igual 4.0 Internacional. Brazilian Journal of Production Engineering, São Mateus, Editora UFES/CEUNES/DETEC. 
Citação (APA): Silva, C. M., da, Silva, M. A. G., da, Pereira, A. S., Franz, L. A. dos S., \& Heidtmann-Bemvenuti, R. (2021). Utilização das tecnologias da indústria 4.0 na segurança e saúde do trabalhador: uma revisão sistemática da literatura. Brazilian Journal of Production Engineering, 7(5), 252-268

da Literatura. Revista Eletrônica de Negócios Internacionais. 16(1), 56-74. Recuperado de https://internext.espm.br/internext/article/view/596

Petz, P., Eibensteiner, F., \& Langer, J. (2021). Sensor Shirt as Universal Platform for RealTime Monitoring of Posture and Movements for Occupational Health and Ergonomics. Procedia Computer Science, 180, 200-207. https://doi.org.10.1016/j.procs.2021.01.157

Pontarolo, M. L. (2018). Relação entre segurança do trabalho e produtividade: uma revisão sistemática. (Trabalho de conclusão de curso de especialização). Universidade Tecnológica Federal do Paraná, Ponta Grossa, PR, Brasil. Recuperado de https://repositorio.utfpr.edu.br/jspui/bitstream/1/23382/1/relacaosegurancatrabalhoprodutivida de.pdf

Rey, R. O., de Melo, R. R. S., \& Costa, D. B. (2021). Design and implementation of a computerized safety inspection system for construction sites using UAS and digital checklists - Smart Inspecs. Safety Science, 143. https://doi.org/10.1016/j.ssci.2021.105430

Rolim, N. R. F., Gabriel, I. de S., Mota, A. S., \& de Quental, O. B. (2020) Fatores que contribuem para a classificação da gestação de alto risco: revisão integrativa. Revista Brasileira de Engenharia de Produção - BJPE. 6(6), 60-68. Recuperado de https://periodicos.ufes.br/bjpe/article/view/31055

Scafa, M., Papetti, A., Brunzini, A., \& Germani, M. (2019). How to improve worker's wellbeing and company performance: a method to identify effective corrective actions. Procedia CIRP, 81, 162-167. https://doi.org/10.1016/j.procir.2019.03.029

Sima, V., Gheorghe, I. G., Subic, J., \& Nancu, D. (2020). Influences of the Industry 4.0 Revolution on the Human Capital Development and Consumer Behavior: A Systematic Review. Sustainability, 12(10), 1-28. https://doi.org/10.3390/su12104035

Steinhilber, B., Seibt, R., Rieger, M. A., \& Luger, T. (2020). Postural Control When Using an Industrial Lower Limb Exoskeleton: Impact of Reaching for a Working Tool and External Perturbation. Human Factors: The Journal of the Human Factors and Ergonomics Society. https://doi.org/10.1177/0018720820957466

Sumer B (2018) Impact of Industry 4.0 on occupations and employment in Turkey. European Scientific Journal, 14(10), 1-17. https://doi.org/10.19044/esj.2018.v14n10p1

Teixeira, R. L. P., Teixeira, C. H. S. B., Brito, M. L. de A., \& Silva, P. C. D. (2019) Os discursos sobre os desafios da siderurgia na indústria 4.0 no Brasil. Brazilian Journal of Development. $5(12)$, 28290-28309. Recuperado de https://www.brazilianjournals.com/index.php/BRJD/article/view/5094

Tessarini, G. \& Saltorato, P. (2018). Impactos da Indústria 4.0 na Organização do Trabalho: Uma Revisão Sistemática da Literatura. Revista Produção Online. 18(2), 743-769. https://doi.org/10.14488/1676-1901.v18i2.2967

Turk, M., Resman, M., \& Herakovic, N. (2021). The impact of smart technologies: A case study on the efficiency of the manual assembly process. Procedia CIRP. 97, 412-417. https://doi.org/10.1016/j.procir.2020.05.260

Vukicevic, A. M., Djapan, M., Stefanovic, M., \& Macuzic, I. (2019). SafE-Tag mobile: A novel javascript framework for real-time management of unsafe conditions and unsafe acts in SMEs. Safety Science, 120, 507-516. https://doi.org/10.1016/j.ssci.2019.07.024 